\title{
Analytical Simulation for Magnetohydrodynamic Maxwell Fluid Flow Past an Exponentially Stretching Surface with First-Order Velocity Slip Condition
}

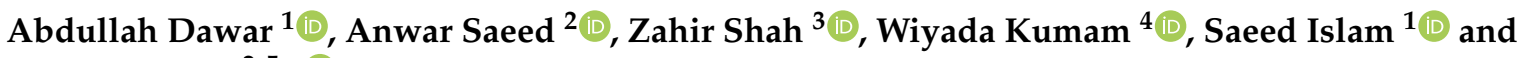 \\ Poom Kumam 2,5,*(D) \\ 1 Department of Mathematics, Abdul Wali Khan University, Mardan 23200, Pakistan; \\ abdullah.mathematician@gmail.com (A.D.); saeedislam@awkum.edu.pk (S.I.) \\ 2 Center of Excellence in Theoretical and Computational Science (TaCS-CoE), Faculty of Science, \\ King Mongkut's University of Technology Thonburi (KMUTT), 126 Pracha Uthit Rd., Bang Mod, \\ Bangkok 10140, Thailand; anwarsaeed769@gmail.com \\ 3 Department of Mathematical Sciences, University of Lakki Marwat, Lakki Marwat 28420, Pakistan; \\ zahir1987@yahoo.com \\ 4 Program in Applied Statistics, Applied Mathematics for Science and Engineering Research Unit (AMSERU), \\ Department of Mathematics and Computer Science, Faculty of Science and Technology, \\ Rajamangala University of Technology Thanyaburi, Pathumthani 12110, Thailand; wiyada.kum@rmutt.ac.th \\ 5 Department of Medical Research, China Medical University Hospital, China Medical University, \\ Taichung 40402, Taiwan \\ * Correspondence: poom.kum@kmutt.ac.th
}

check for updates

Citation: Dawar, A.; Saeed, A.; Shah, Z.; Kumam, W.; Islam, S.; Kumam, P. Analytical Simulation for

Magnetohydrodynamic Maxwell Fluid Flow Past an Exponentially Stretching Surface with First-Order Velocity Slip Condition. Coatings 2021, 11, 1009. https://doi.org/10.3390/ coatings11081009

Academic Editor: Rahmat Ellahi

Received: 2 August 2021

Accepted: 18 August 2021

Published: 23 August 2021

Publisher's Note: MDPI stays neutral with regard to jurisdictional claims in published maps and institutional affiliations.

Copyright: (c) 2021 by the authors. Licensee MDPI, Basel, Switzerland. This article is an open access article distributed under the terms and conditions of the Creative Commons Attribution (CC BY) license (https:/ / creativecommons.org/licenses/by/ $4.0 /)$.

\begin{abstract}
The study of fluid flow upon an exponentially stretching surface has significant importance due to its applications in technological phenomena at the industrial level. These applications include condensing process of fluid film, heat exchanger processes, extrusion of plastic sheet in aerodynamics, cooling process of metal sheet, and growth of crystals, etc. Keeping in view all these applications, in this paper, we have discussed the magnetohydrodynamic flow of Maxwell fluid past an exponentially stretching sheet. The stretching surface is considered to be slippery by imposing the velocity slip condition. The magnetic field impact is taken into consideration. Furthermore, heat radiation, Joule heating, Brownian motion, and thermophoresis are also considered. The modeled system is reduced to ordinary differential equations with the help of similarity variables. For the analytical solution, we have used the homotopy analysis method. Furthermore, HAM is compared with the shooting method and found to be in great agreement. The squared residual error of the fluid flow problem at 15th order of approximations for Newtonian and non-Newtonian cases has been investigated. It is found that the fluid flow problem converges quickly for the case of non-Newtonian fluid as compared to Newtonian fluid. In addition, the velocity profile increases while the thermal and concentration profiles reduce with greater values of Darcy number. The thermal profile is the increasing function of the Brownian motion parameter and Eckert number whereas the concentration profile is the reducing function of the Brownian motion parameter and Eckert number. With the augmentation in Darcy number, the permeability strength of porous medium increases which concludes the increasing conduct of thermal and mass transportation.
\end{abstract}

Keywords: Maxwell fluid; exponentially stretching sheet; velocity slip condition; porous medium; MHD

\section{Introduction}

The effect of magnetohydrodynamic (MHD) fluid flow is a physical property that describes the movement of an electrically conducted fluid with the impact of externally applied magnetic effects. The salt water, electrolytes, and plasma, etc. are MHD fluids. MHD fluid flow has plentiful industrial applications, for instance, drug targeting and 
cooling of reactors, etc. MHD is based upon the induction of electric current by applied magnetic field through a conducted moving fluid. The idea of MHD was first floated by Swedish electrical engineer Alfven [1], a developer of Alfven's waves. Many investigators have used the concept of MHD in various investigations. Turkyilmazoglu [2] has inspected the thermal characteristics for MHD fluid flow upon stretching a rotary disk by using Joule heating and viscous dissipation in the thermal equations. In this study, it has been noticed that an augmentation in magnetic effects has opposed the flow of fluid and has supported the thermal flow. Kumar et al. [3] investigated the influences of thermal radiations upon MHD fluid flow over an exponential stretched sheet. A comparison has been accumulated in this work with a fine agreement between the claimed and established results. Khan et al. $[4,5]$ have established incredible results for MHD fluid flows and heat transfers by employing different flow conditions. The authors have proven that the augmented values of the magnetic field have enhanced the transmission of heat while they have decreased the flow behavior.

The fluid that exhibits viscoelastic characteristics, which is fluid with the behavior of viscosity as well as elasticity, is termed as Maxwell fluid. The Maxwell fluid model was proposed first by James Clerk Maxwell [6]. Due to its importance, many researchers have used Maxwell fluid in the investigation of mass and heat transfer. Islam et al. [7] inspected the impact of thermal radiations on Maxwell nanoparticle flow upon a stretching cylinder. Semi-numerical technique has been used in the study and has established that due to enhancement in Maxwell parameter, the stress relaxation phenomenon has been augmented, due to which the flow of fluid has declined. Shehzad et al. [8] have discussed the forced convection Maxwell fluid flow with the impact of thermophoresis particle movements past a spinning disk. Khan et al. [9] have analyzed the MHD Maxwell fluid flowing on the stretched surface. In this examination, the heat and mass transmission have been investigated by using the impacts of variations in thermal conductivity and the Cattaneo-Christove effects. It has been noticed in this work that, with an augmentation in Deborah number, more resistance has been offered to the fluid flow particles that have decreased the flow and concentration of fluid and have enhanced the thermal characteristics. Ahmad et al. [10] have inspected the unsteady Maxwell fluid flow past a time-dependent stretching sheet and have established that augmented values of Brownian motion have jumped up the thermal profile and have jumped down the flow and concentration of fluid. Farooq et al. [11] inspected the MHD and Maxwell fluid flow over the exponential stretched surface by using the famous Boungiorno model.

The study of fluid flow upon the exponentially stretching surface is of significant importance due to its applications in technological phenomena at the industrial level. These applications comprise condensing process of fluid film, heat exchanger processes, extrusion of plastic sheet in aerodynamics, cooling process of metal sheet, and growth of crystals, etc. After the revolutionary work of Sakiadis [12,13], many investigators have carried out different examinations for fluid flow upon exponentially stretching surfaces with a prime focus upon the mass and thermal flow. Nadeem et al. [14] have discussed the influences of slip effects over nanoparticle flow over a stretching sheet. The authors of this investigation have observed that with augmentation of stretching parameter, the microorganism and Nusselt numbers have enhanced while the skin fraction has declined with this increase. Lund et al. [15] examined the MHD fluid flow upon an exponentially stretching surface with the suction condition. Waini et al. [16] have inspected the mixed convective flow of hybrid nanofluid over an exponentially stretched medium and have established that the solid nanoparticles have decreased the flow profiles and have increased the thermal transmission. Patil et al. [17] inspected the influences of roughness upon mixed convection nanoparticle flow over an exponentially stretched surface. It has been observed in this work that the thermal flow rate of the wall is considerably controlled by the addition of nanoparticles. The topic can be further studied in the Refs. [18-23].

A surface that contains void spaces is termed a porous surface such as rubber, some rocks, woods, and sponges, etc., which are available in nature. Normally, a porous surface 
is described by the porosity of that surface. Some porous surfaces are manmade, such as ceramics and cements, etc. There are various applications of porous media in applied sciences and engineering, for instance, rock mechanics, geomechanics, petroleum geology, geophysics, and biophysics, etc. Because of its importance, many investigations have been conducted by different researchers. Alazmi and Vafai [24] have analyzed the thermal and fluid flow upon a porous medium in the combination of the fluid layer. Fatunmbi et al. [25] have discussed micropolar MHD fluid flow past a porous medium using slip conditions. It has been observed in this work that augmentation in stretching parameter has adverse effects upon the flow and thermal profiles. Krishna et al. [26] have inspected the time-dependent MHD flow of micropolar fluid upon permeable surface using the influence of ion slip and Hall current. In this investigation, the effects of Sherwood and Nusselt numbers have been investigated numerically with the influence of different physical parameters. Hayat et al. [27] have inspected the time-dependent nanoparticle fluid flow upon a permeable surface using different flow conditions and characteristics. In this investigation, Darcy-Forchheimer and Boungiorno's models have also been incorporated by the authors in the flow problem. It has been noticed in this study that augmented values of Darcy number and inertia parameters have enhanced the thermal profiles and have decreased the flow profiles. These same effects have also been observed for augmentation in Brownian effects. Asjad et al. [28] inspected the MHD fluid flow in a permeable medium with the help of fractional order derivatives. The authors have used the transform method to determine the exact solution. Khan et al. [29] have inspected the generation of entropy for thermos-solutal convection problems over porous medium. In this study, a newer mass Bejan number has been described for the first time using two salt concentrations. Jafar et al. [30] discussed MHD nanoparticle flow over a permeable and stretching surface with thermal radiation. The authors have used the Keller Box technique for the approximate solution of the problem.

In light of the above literature review, we are interested to investigate the Maxwell fluid flow through an exponentially stretching surface with first-order velocity slip condition using the porous medium. The homotopy analysis method is employed in order to investigate the proposed model analytically. Furthermore, heat radiation, Joule heating, Brownian motion, and thermophoresis are also considered.

\section{Problem Formulation}

Consider a laminar, two-dimensional, and incompressible MHD Maxwell fluid flow past an exponentially stretching sheet with first-order velocity slip condition. The flow of Maxwell fluid is submerged in a porous medium. The stretching velocity of the surface is considered as $U_{w}=U_{0} \exp (x / L)$ along $x$ - direction, whereas $y$-direction is perpendicular to the stretching sheet. Furthermore, the heat radiation, Joule heating, Brownian motion, and thermophoresis phenomena are also considered.

In view of the above assumptions, the leading equations take the form:

$$
\begin{gathered}
u \frac{\partial T}{\partial x}+v \frac{\partial T}{\partial y}=\alpha \frac{\partial^{2} T}{\partial y^{2}}+\frac{v}{c_{p}}\left(\frac{\partial u}{\partial y}\right)^{2}+\left(\frac{\sigma B_{0}^{2}}{\rho c_{p}}+\frac{v}{K c_{p}}\right) u^{2}+\frac{1}{\rho c_{p}} \frac{\partial q_{r}}{\partial y}+\frac{Q}{\rho c_{p}}\left(T-T_{\infty}\right)+\tau\left(D_{B} \frac{\partial T}{\partial y} \frac{\partial C}{\partial y}+\frac{D_{T}}{T_{\infty}}\left(\frac{\partial T}{\partial y}\right)^{2}\right) \\
u \frac{\partial C}{\partial x}+v \frac{\partial C}{\partial y}=D_{B} \frac{\partial^{2} C}{\partial y^{2}}+\frac{D_{T}}{T_{\infty}} \frac{\partial^{2} T}{\partial y^{2}}
\end{gathered}
$$


where $u$ and $v$ are the velocity components, $\rho$ is the fluid density, $B_{0}$ is the strength of magnetic field, $\lambda$ is the relaxation time, $q_{r}=-4 \sigma^{*} / 3 k^{*}\left(\partial T^{4} / \partial y\right)$ is the radiative heat flux, $K$ is the permeability, $c_{p}$ is the specific heat, $\alpha=k / \rho c_{p}$ is the thermal diffusivity, $D_{B}$, $D_{T}$ are the Brownian and thermophoresis coefficients, $U_{0}$ is reference velocity, $T$ is the temperature, $T_{0}, T_{\infty}$ are the reference and ambient temperatures, $C$ is the concentration, $C_{0}$ is the reference concentration, and $C_{\infty}$ is the ambient concentration.

The boundary conditions are defined as:

$$
\left\{\begin{array}{l}
u=U_{w}+\frac{\mu}{L_{1}} \frac{\partial u}{\partial y}, v=0, C=C_{w}, T=T_{w} \text { at } y=0, \\
u \rightarrow 0, C \rightarrow C_{\infty}, T \rightarrow T_{\infty} \text { as } y \rightarrow \infty .
\end{array}\right\}
$$

Here, $\mu$ is the viscosity coefficient, $L_{1}$ is the slip length, $T_{w}=T_{\infty}+T_{0} \exp (x / 2 L)$ and $C_{w}=C_{\infty}+C_{0} \exp (x / 2 L)$ are the varying temperature and concentration.

The similarity variables are defined as:

$$
\begin{aligned}
& u=U_{0} \exp \left(\frac{x}{L}\right) f^{\prime}(\xi), v=-\sqrt{\frac{v U_{0}}{2 L}} \exp \left(\frac{x}{2 L}\right)\left(f(\xi)+\xi f^{\prime}(\xi)\right), \theta(\xi)=\frac{T-T_{\infty}}{T_{0}} \exp \left(-\frac{x}{2 L}\right), \\
& \phi(\xi)=\frac{C-C_{\infty}}{C_{0}} \exp \left(-\frac{x}{2 L}\right), \xi=y \sqrt{\frac{U_{0}}{2 v L}} \exp \left(\frac{x}{2 L}\right), \psi=\sqrt{2 v L U_{0}} \exp \left(\frac{x}{2 L}\right) f(\xi) .
\end{aligned}
$$

where $L$ is the reference length.

Using the above similarity transformations, Equation (1) is obvious and Equations (2)-(5) are transformed as:

$$
\begin{gathered}
f^{\prime \prime \prime}+f f^{\prime \prime}-2 f^{2}+\beta\left(\frac{\xi}{2} f^{\prime 2} f^{\prime \prime}+3 f f^{\prime} f^{\prime \prime}-2 f^{\prime 3}-\frac{1}{2} f^{2} f^{\prime \prime \prime}\right)-\left(M+\frac{1}{D a}\right) f^{\prime}=0, \\
(1+R d) \theta^{\prime \prime}+\operatorname{Pr}\left(f \theta^{\prime}-f^{\prime} \theta+E c\left(M+\frac{1}{D a}\right) f^{\prime 2}+E c f^{\prime \prime}{ }^{2}+2 S \theta+N b \theta^{\prime} \phi^{\prime}+N t \theta^{\prime 2}\right)=0, \\
\phi^{\prime \prime}+\frac{N t}{N b} \theta^{\prime \prime}+\operatorname{Pr} L e\left(f \phi^{\prime}-f^{\prime} \phi\right)=0, \\
f(0)=0, f^{\prime}(0)=1+\lambda_{1} f^{\prime \prime}(0), \theta(0)=1, \phi(0)=1, f^{\prime}(\infty)=0, \theta(\infty)=0, \phi(\infty)=0 .
\end{gathered}
$$

In the above system of equations, $\beta=\lambda U_{w} / L$ is the Maxwell parameter, Prandtl number is $\operatorname{Pr}=\mu c_{p} / k, M=2 L \sigma B_{0}^{2} / \rho U_{w}$ is the magnetic parameter, $N t=\tau D_{T}\left(T_{w}-T_{\infty}\right) / v T_{\infty}$ and $\mathrm{Nb}=\tau D_{B}\left(C_{w}-C_{\infty}\right) / v$ are thermophoresis and Brownian parameters, the Lewis number is $L e=v / D_{B}, D a=K U_{w} / 2 v L$ is the Darcy's number, $E c=U_{w}^{2} / c_{p} T_{w}$ is the Eckert number, $S=Q L / U_{w} c_{p}$ is heat source/sink parameter, and thermal radiation parameter is $R d=16 \sigma^{*} T_{\infty}^{3} / 3 k k^{*}$.

The quantities of engineering interest are described as:

$$
\left\{\begin{array}{c}
N u_{x}=-\left.\frac{x(1+R d)}{T_{w}-T_{\infty}} \frac{\partial T}{\partial y}\right|_{y=0}, \\
S h_{x}=-\left.\frac{x}{C_{w}-C_{\infty}} \frac{\partial C}{\partial y}\right|_{y=0} ^{\cdot}
\end{array}\right\}
$$

In view of Equation (6), Equation (11) is reduced as:

$$
\left\{\begin{array}{l}
\frac{N u_{x}}{\sqrt{\frac{x \mathrm{Re}_{x}}{2 L}}}=-(1+R d) \theta^{\prime}(0), \\
\frac{S h_{x}}{\sqrt{\frac{x \mathrm{Re}_{x}}{2 L}}}=-\phi^{\prime}(0),
\end{array}\right\}
$$

where $\operatorname{Re}_{x}=U_{w} x / v$ describes the local Reynolds number. 


\section{HAM Solution}

In order to solve Equations (7)-(9) along with the respective boundary conditions defined in Equation (10), homotopy analysis method is applied. The initial guesses and linear operators are defined as:

$$
\begin{gathered}
\left\{\begin{array}{l}
f_{0}(\xi)=\frac{1}{1+\lambda_{1}}(1-\exp (-\xi)), \\
\theta_{0}(\xi)=\exp (-\widetilde{\xi}) \\
\phi_{0}(\xi)=\exp (-\xi)
\end{array}\right\} \\
\left\{\begin{array}{c}
L_{f}=\frac{\partial^{3} f}{\partial \xi^{3}}-\frac{\partial f}{\partial \xi^{\prime}} \\
L_{\theta}=\frac{\partial^{2} \theta}{\partial \xi^{2}}-\theta \\
L_{\phi}=\frac{\partial^{2} \phi}{\partial \xi^{2}}-\phi
\end{array}\right\}
\end{gathered}
$$

with the properties:

$$
\left\{\begin{array}{l}
L_{f}\left(x_{1}+x_{2} \exp (-\xi)+x_{3} \exp (\xi)\right)=0 \\
L_{\theta}\left(x_{4} \exp (-\xi)+x_{5} \exp (\xi)\right)=0 \\
L_{\phi}\left(x_{6} \exp (-\xi)+x_{7} \exp (\xi)\right)=0
\end{array}\right\}
$$

where $x_{i}(i=1-7)$ are the arbitrary constant.

\section{Results and Discussion}

The purpose of this section is to examine the impact of embedding factors on different profiles of the flow system. The current study's findings are presented graphically. It should be noted that we have chosen some suitable values for emerging parameters like $\beta=0.1, M=0.2, D a=0.5, R d=0.3, \operatorname{Pr}=1.0, E c=0.1, S=0.1, N b=2.0, N t=0.5$, $L e=1.0$, and $\lambda=0.5$.

Figures 1-3 show the effect of Darcy number on velocity, thermal, and concentration profiles, respectively. The velocity profile augments while the thermal and concentration profiles reduce with the greater Darcy number. Darcy number is the characterization of the permeability strength of the porous medium. As we increase the Darcy number, the permeable strength of the porous medium becomes greater, which consequently increases the velocity profile. However, the layers of thermal and concentration boundaries reduce with the greater Darcy number. Thus, the thermal and concentration profiles are the reducing functions of the Darcy number. Figure 4 shows the effect of Deborah number on velocity profile. The greater Deborah number reduces the velocity profile. The maximum influence of Deborah number is perceived at the surface of the fluid flow; however, this impact reduces gradually, and the properties of the non-Newtonian fluid shift to Newtonian fluid. Thus, as we increase the Deborah number, the velocity profile reduces. Figures 5-7 depict the impact of magnetic field on velocity, thermal, and concentration profiles, respectively. The velocity profile reduces while the thermal and concentration profiles augment with a greater magnetic field parameter. The greater magnetic field parameter yields to the Lorentz force which always opposes the flow of fluid. Thus, a reducing impact is observed here. Furthermore, the temperature and mass profiles are the augmenting functions of the magnetic field. Figures 8 and 9 portray the impact of thermophoretic parameters on thermal and concentration flow profiles. Both the thermal and concentration profiles are increasing functions of thermophoresis parameter. This behavior is because the greater thermophoresis parameter moves the nanoparticles of fluid from hot to colder region which results in increasing the temperature of the fluid flow. Thus, an increasing impact of thermophoresis parameter on thermal profile is depicted here. A similar impact of thermophoresis parameter on concentration profile is observed. Figures 10 and 11 show the influence of Brownian motion on thermal and concentration 
profiles, respectively. Brownian motion is responsible for augmentation in thermal profile and a reduction in concentration profile. The greater Brownian motion parameter heats up the concentration boundary layer thickness, which moves the fluid nanoparticles from the exponentially extending surface to motion less fluid. Therefore, the concentration profile reduces with greater Brownian motion parameter. Figures 12 and 13 signify the outcome of Eckert number on thermal and concentration profiles. The thermal profile is the increasing function, while the concentration profile is the reducing function of the Eckert number. Eckert number is mostly utilized in nuclear engineering systems for compressible flow at high speeds. The increasing Eckert number heightens the internal energy which consequently increases the temperature of the fluid. A very slight diminishing influence of Eckert number on concentration profile is depicted. Figure 14 shows the impact of Lewis number on concentration profile. The greater Lewis number reduces the concentration profile. The greater Brownian motion diffusion coefficient is responsible for the reduction in concentration of the fluid flow because the Brownian motion diffusion coefficient is inversely related to Lewis number. Thus, the reducing impact in concentration profile is depicted. Figures 15-17 demonstrate the comparison of HAM and numerical methods for velocity, thermal, and concentration functions respectively. Figures $18-20$ show the squared residual error of the fluid flow problem at 15th order of HAM for Newtonian $(\beta=0.0)$ and non-Newtonian $(\beta=0.5$ and $\beta=1.0)$ cases. The fluid flow problem converges quickly for the case of non-Newtonian fluid when $\beta=1.0$ as compared to Newtonian fluid. Table 1 shows the comparison of the current analysis with previous studies. Here, we have found great agreement of our current study with previously published results. Table 2 shows the squared residual errors along with CPU time for velocity, temperature, and concentration profiles. Tables 3 and 4 show the impacts of different embedded factors on thermal and mass transfer rates. It is observed that the radiation parameter augments the values of $N u_{x}$ and $S h_{x}$. It is obvious that the higher thermal radiation escalates the heat transfer rate while an opposite impact is observed against mass transfer rate. The heat transfer rate is a declining function of $\mathrm{Nb}$ and $\mathrm{N} t$. As a result, both Brownian motion and thermophoresis processes in the current work tend to push the nanoparticles away from the stretching surface. Moreover, increasing Brownian motion or thermophoresis parameters accelerates the nanoparticles' diffusion into the boundary layer, lowering the heat transfer rate. Moreover, a decreasing impact of $\mathrm{Nb}$ on mass transfer rate is observed while the thermophoresis parameter has increasing impact on mass transfer rate. It is observed that the higher Darcy number augments both these rates. Actually, the greater values of $D a$ designates the porousness of porous media. Therefore, as we increase the Darcy number, the permeability strength of porous media increases, which concludes the increasing conduct in transportation rates of mass and heat. It is perceived that the higher magnetic parameter jumps down $N u_{x}$ and jumps up $S h_{x}$. Physically, the addition of magnetic effects to the fluid flow creates the resistive force at the surface of the fluid flow which raises the skin friction, but at the same time, the heat transfer rate reduces. In addition, it is found that $S h_{x}$ augmenting function of magnetic parameter is at the surface of the fluid while after some time the mass transfer rate is declined. It has been noticed that the higher Eckert number reduces the heat and mass transfer rates. Actually, the greater Eckert number increases the thermal and nanoparticle boundary layer thicknesses, which consequently reduce the thermal and mass transportation rates. Tables $5-7$ show the comparison of HAM and numerical method for velocity, temperature, and concentration profiles, respectively. These tables are associated with Figures 15-17. 


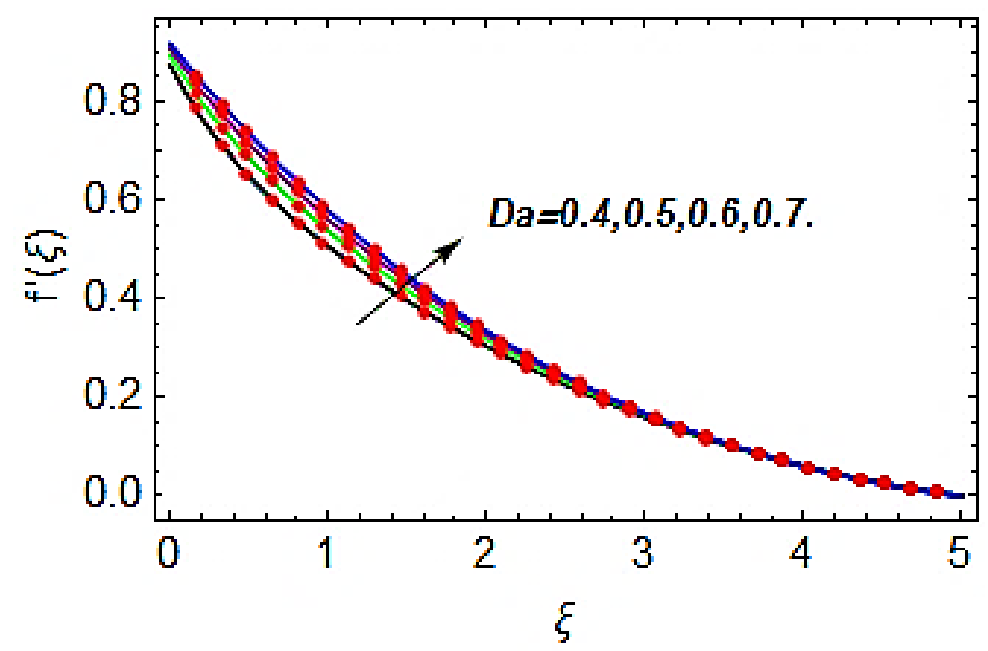

Figure 1. Impact of $D a$ on $f^{\prime}(\xi)$.

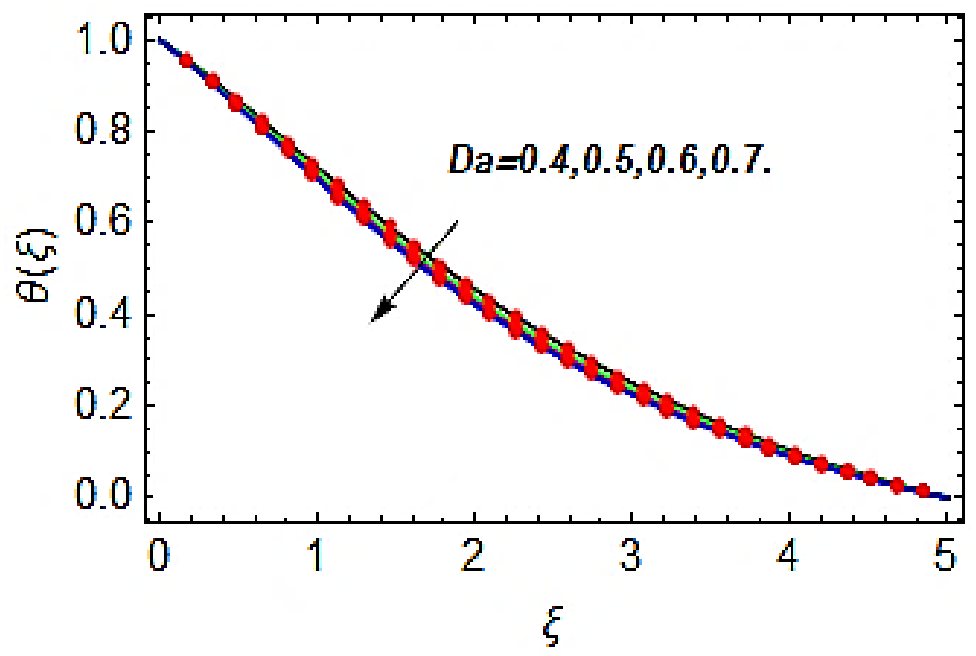

Figure 2. Impact of $D a$ on $\theta(\xi)$.

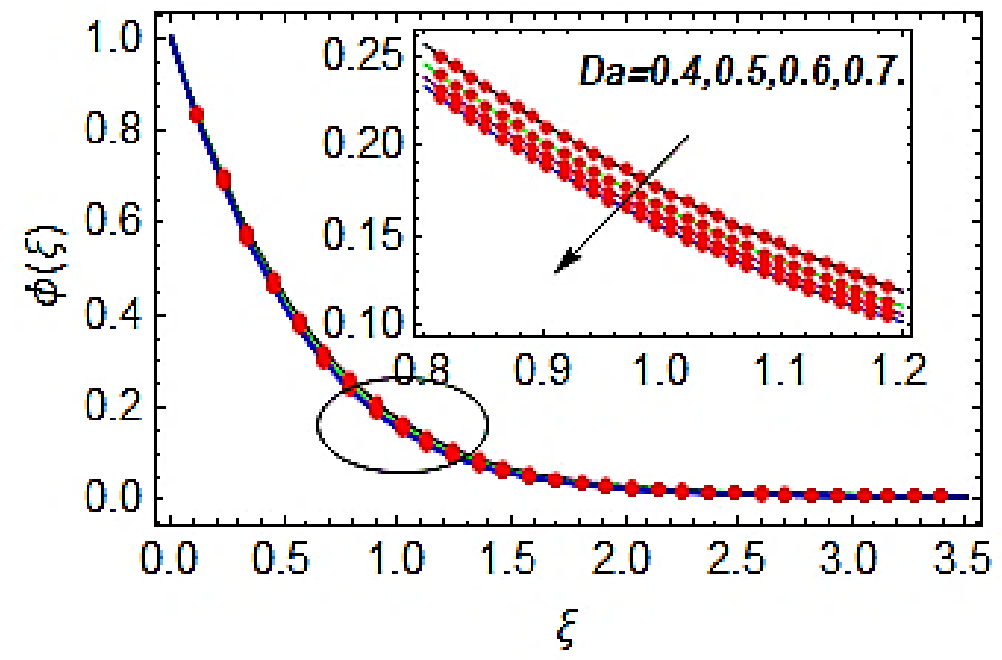

Figure 3. Impact of $D a$ on $\phi(\xi)$. 


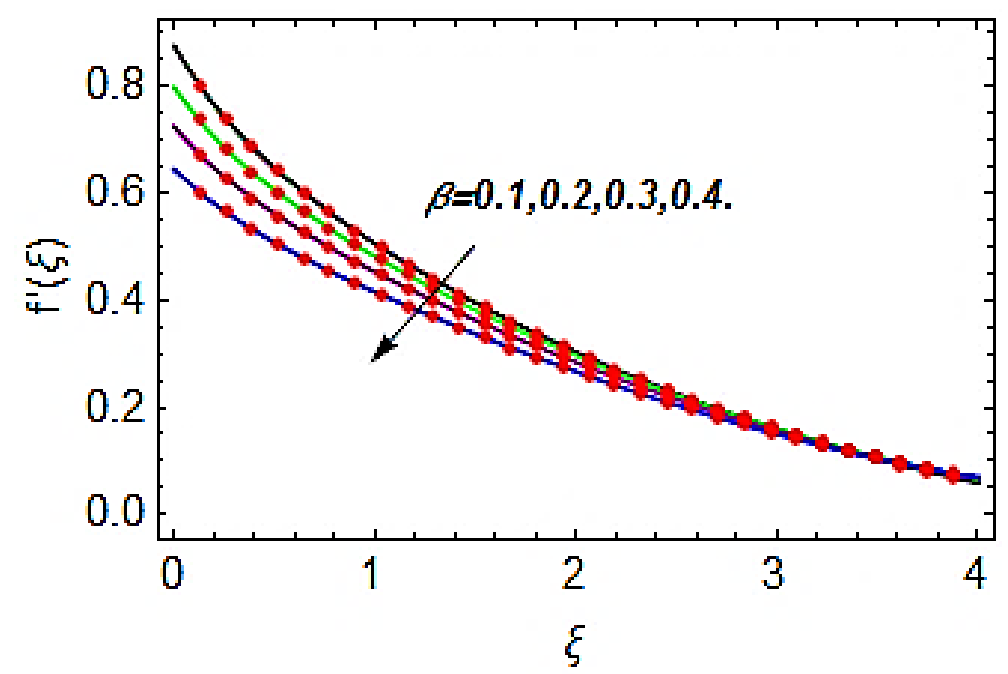

Figure 4. Impact of $\beta$ on $f^{\prime}(\xi)$.

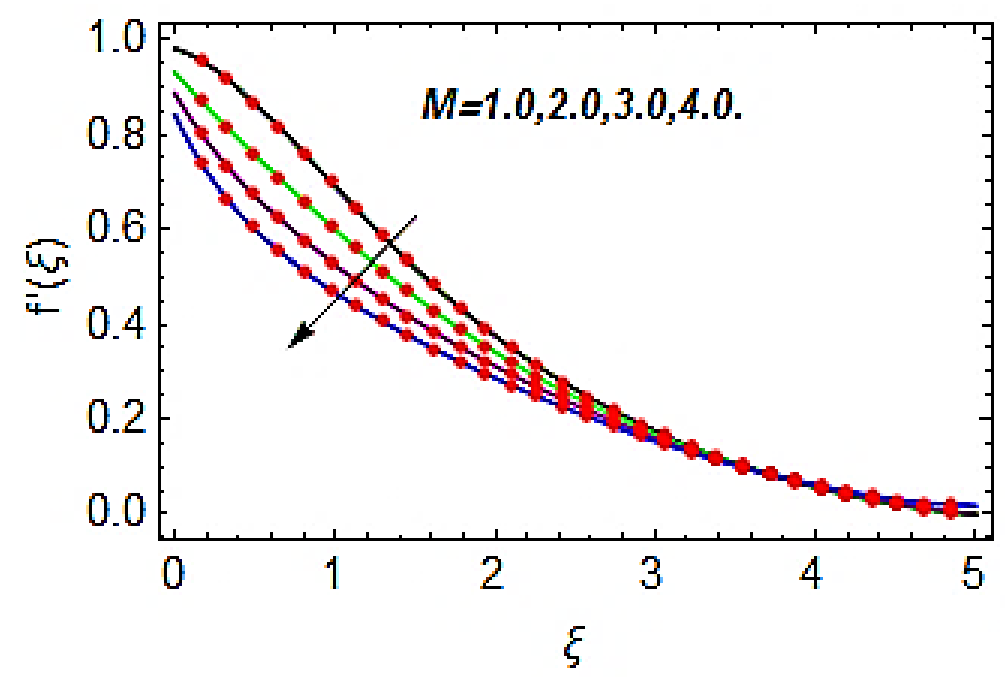

Figure 5. Impact of $M$ on $f^{\prime}(\xi)$.

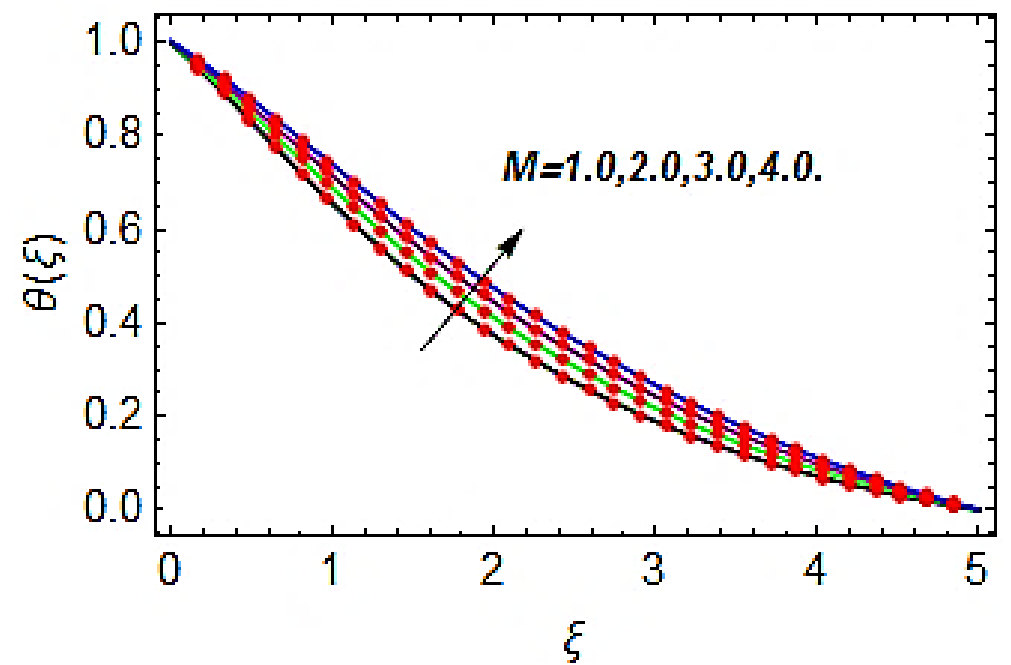

Figure 6. Impact of $M$ on $\theta(\xi)$. 


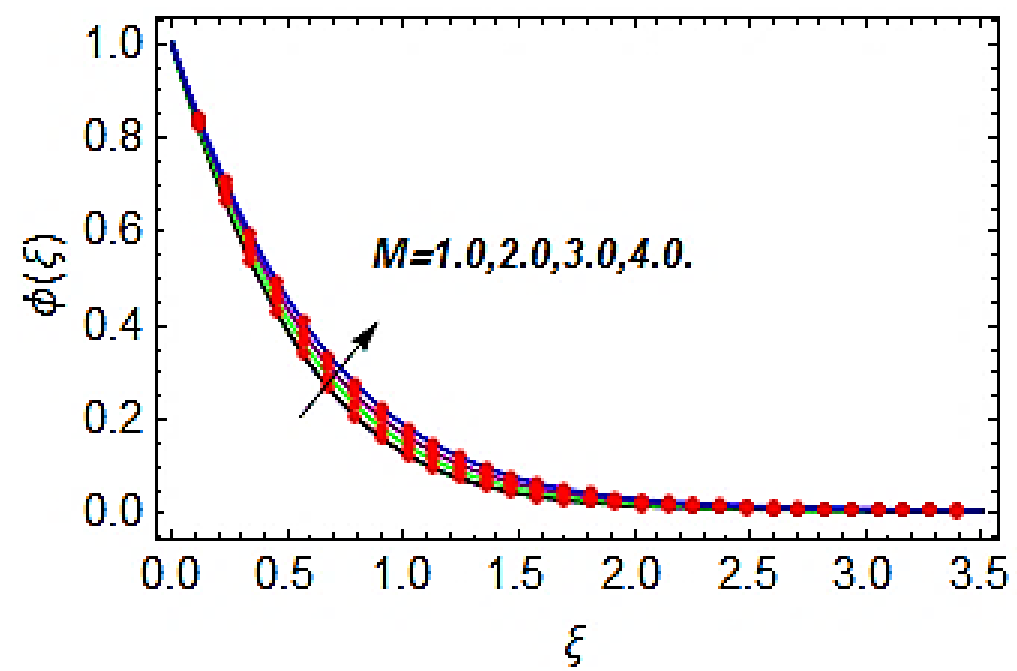

Figure 7. Consequence of $M$ on $\phi(\xi)$.

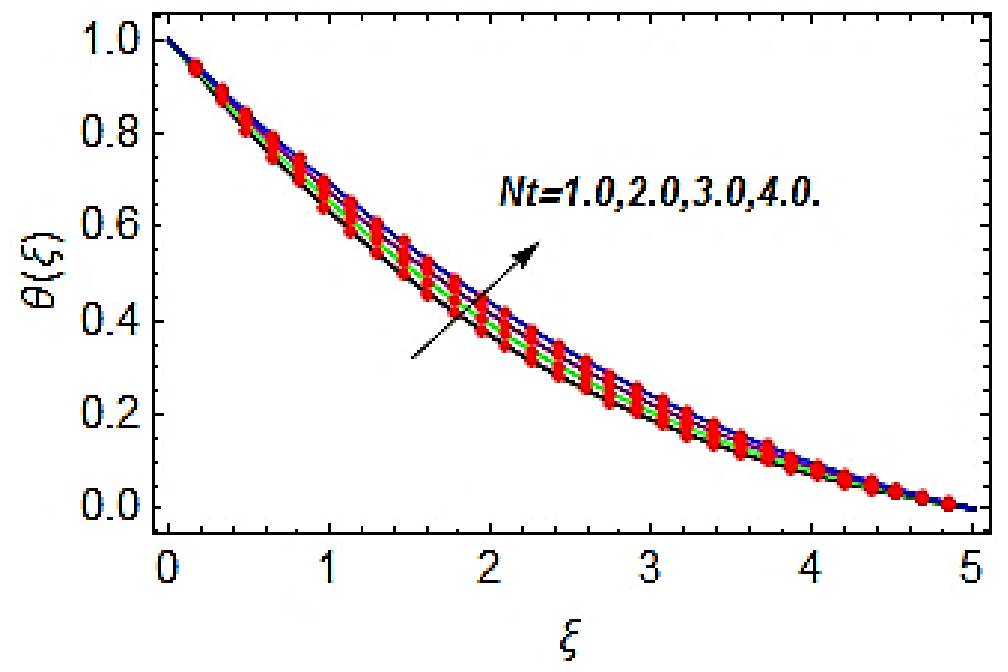

Figure 8. Impact of $N t$ on $\theta(\xi)$.

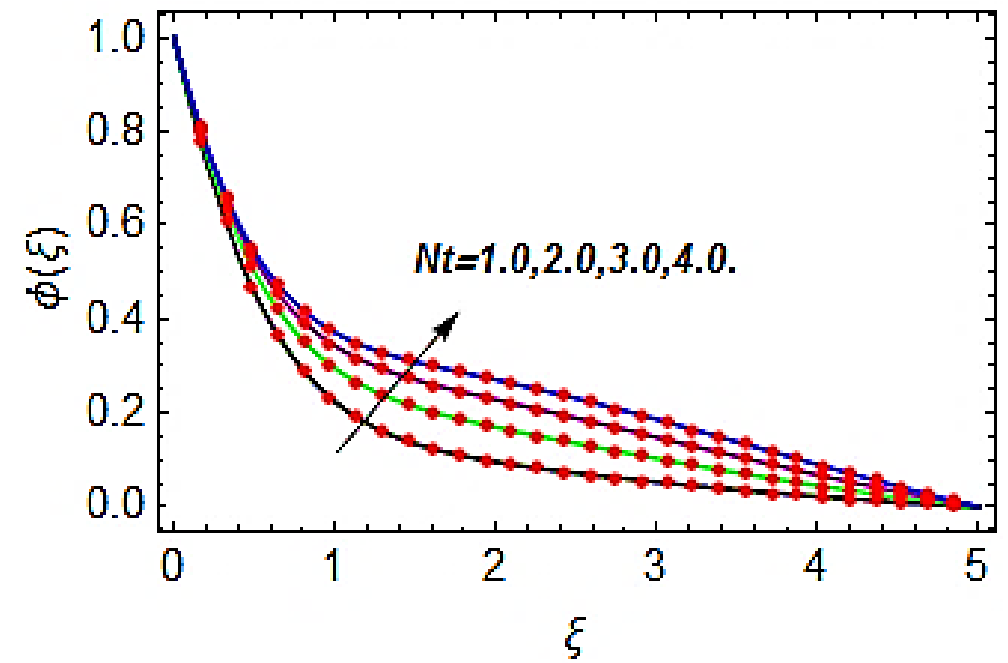

Figure 9. Impact of $N t$ on $\phi(\xi)$. 


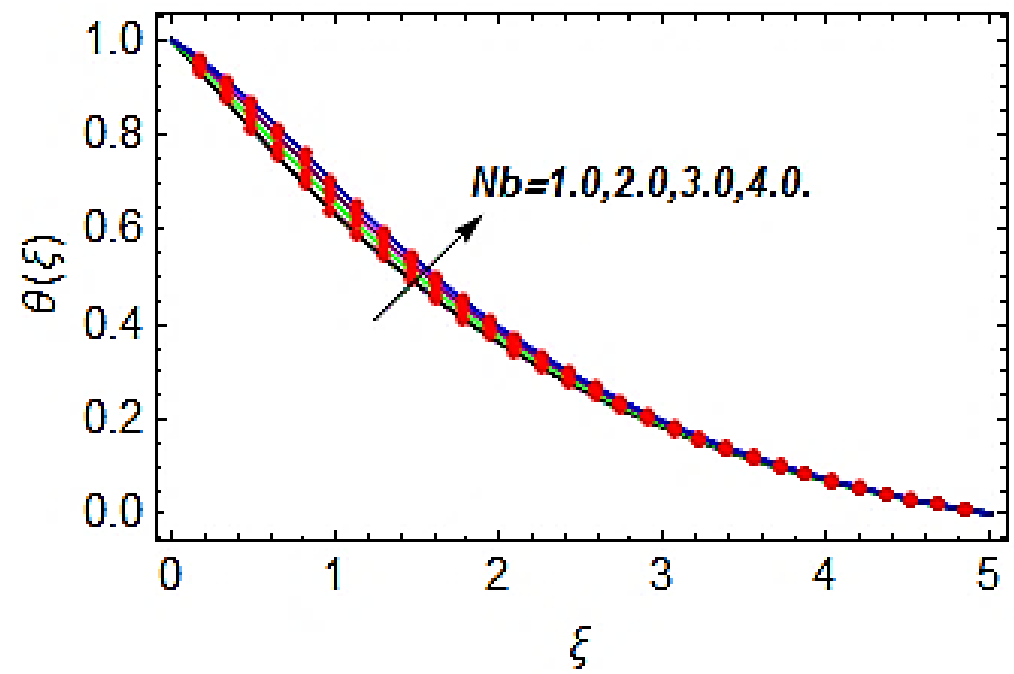

Figure 10. Impact of $N b$ on $\theta(\xi)$.

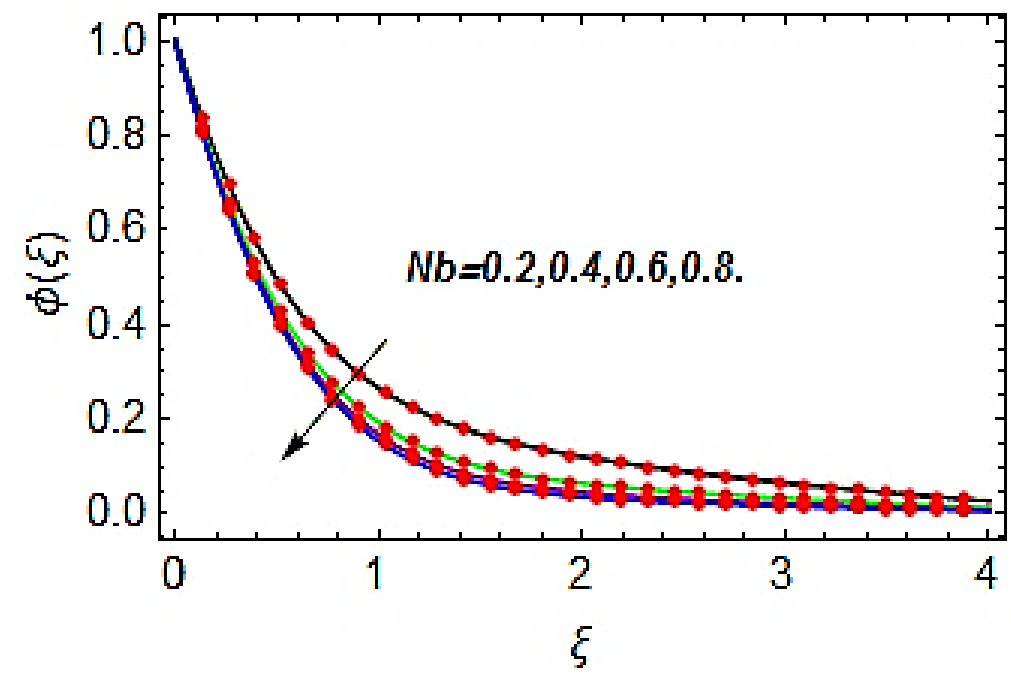

Figure 11. Impact of $N b$ on $\phi(\xi)$.

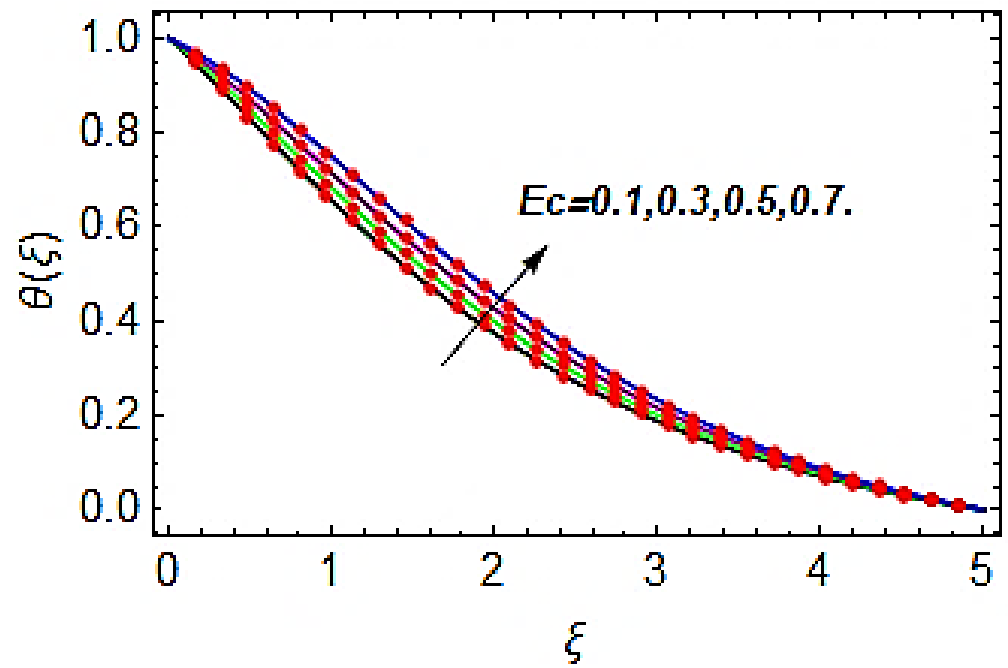

Figure 12. Impact of $E_{c}$ on $\theta(\xi)$. 


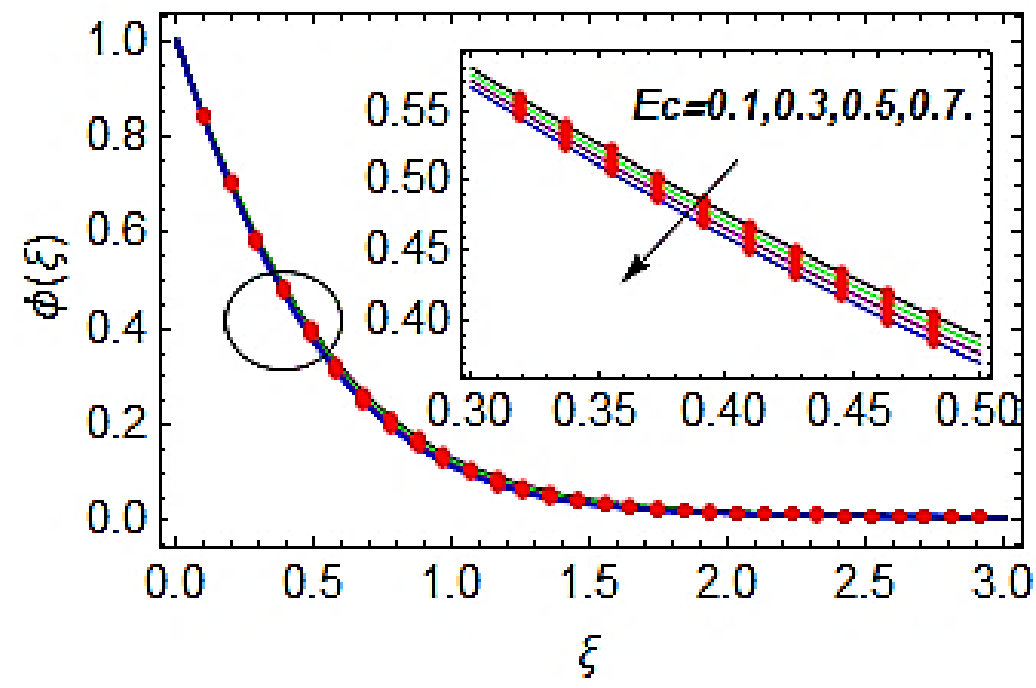

Figure 13. Impact of $E c$ on $\phi(\xi)$.

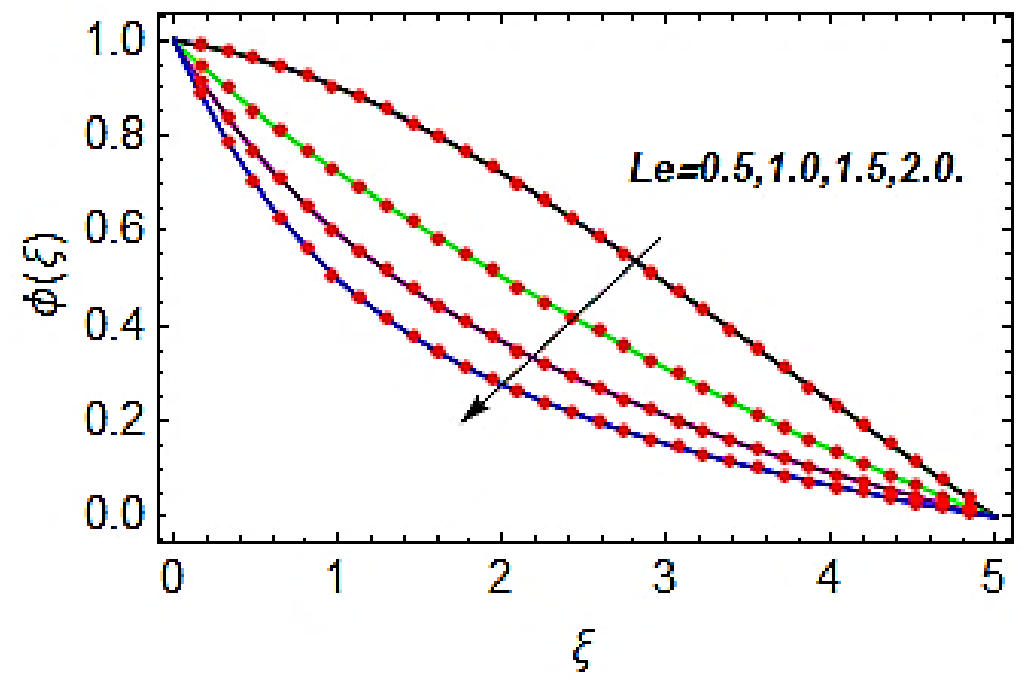

Figure 14. Impact of Le on $\phi(\xi)$.

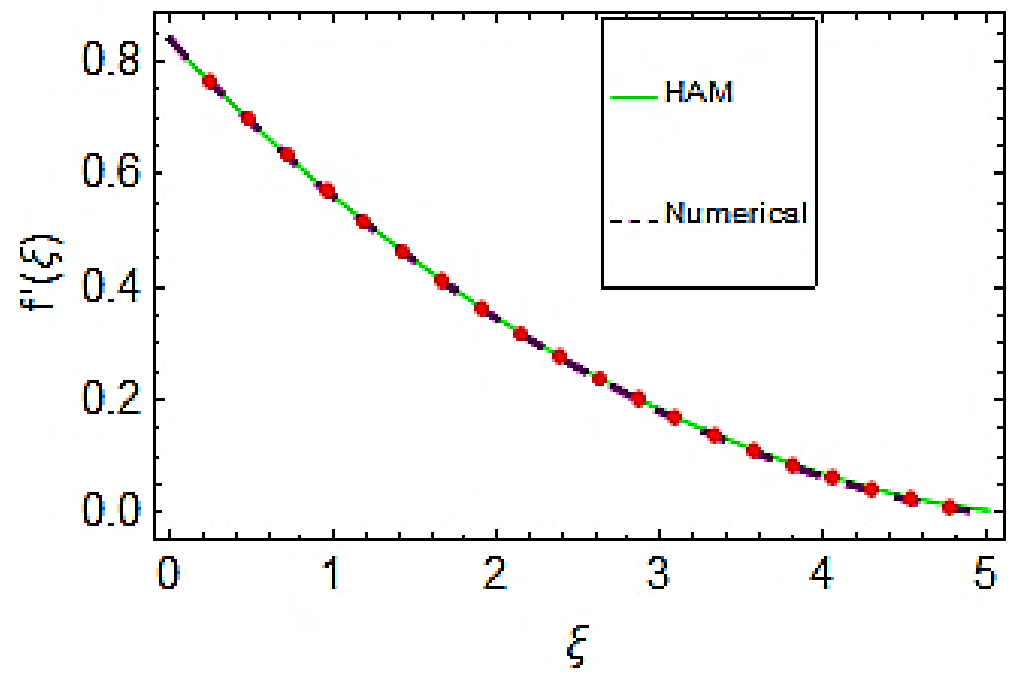

Figure 15. Assessment of HAM and numerical method for $f^{\prime}(\xi)$. 


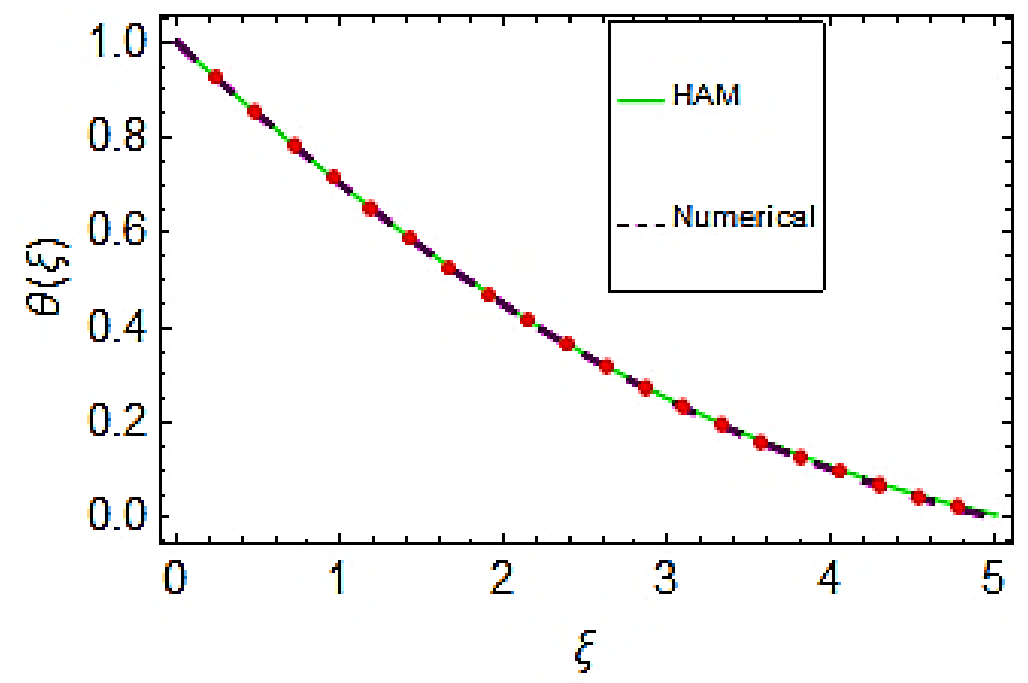

Figure 16. Assessment of HAM and numerical method for $\theta(\xi)$.

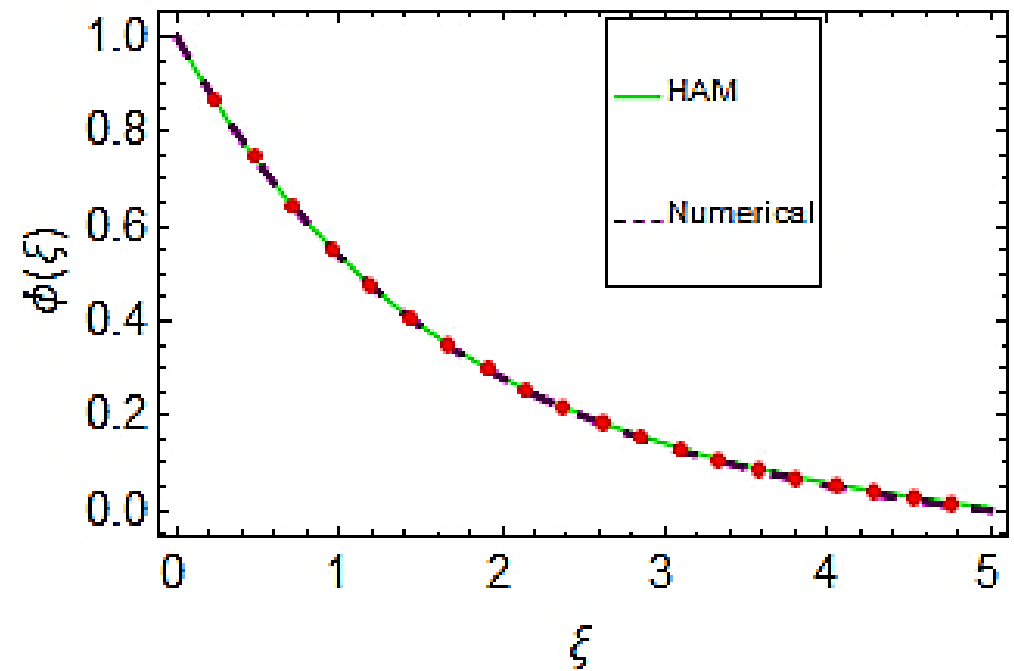

Figure 17. Assessment of HAM and numerical method for $\phi(\xi)$.

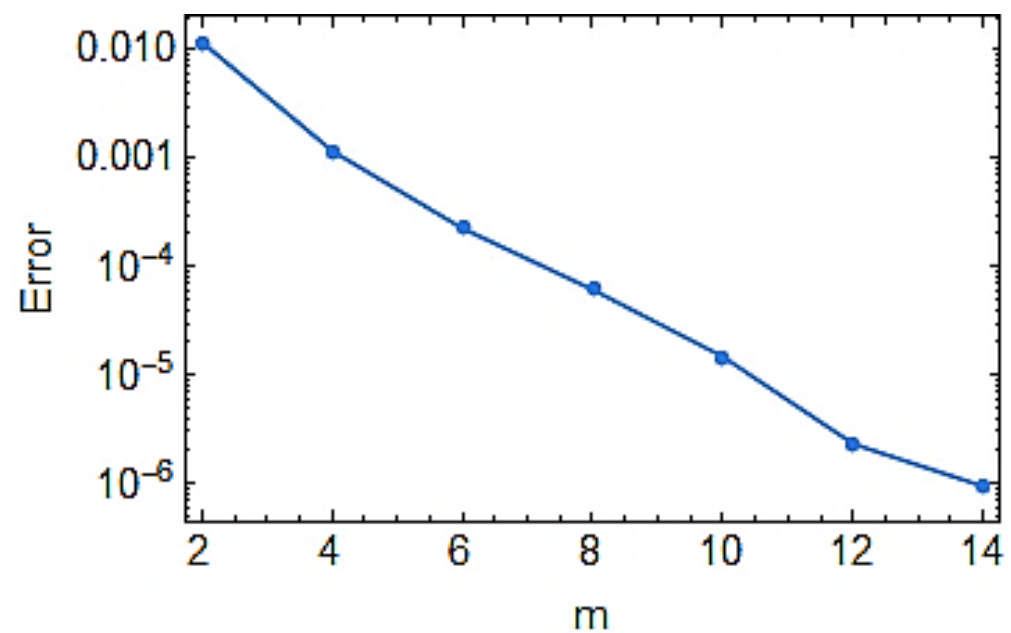

Figure 18. Error of the fluid flow problem at 15th order of HAM when $\beta=0.0, M=0.2, D a=0.5$, $R d=0.3, \operatorname{Pr}=1.0, E c=0.1, S=0.1, N b=2.0, N t=0.5, L e=1.0$, and $\lambda=0.5$. 


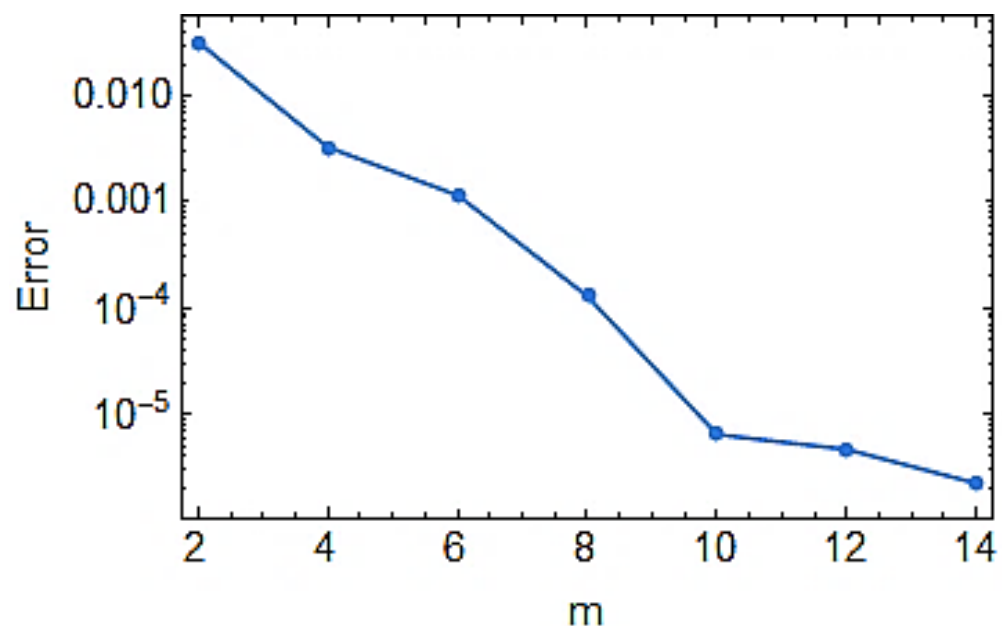

Figure 19. Error of the fluid flow problem at 15th order of HAM when $\beta=0.5, M=0.2, D a=0.5$, $R d=0.3, \operatorname{Pr}=1.0, E c=0.1, S=0.1, N b=2.0, N t=0.5, L e=1.0$, and $\lambda=0.5$.

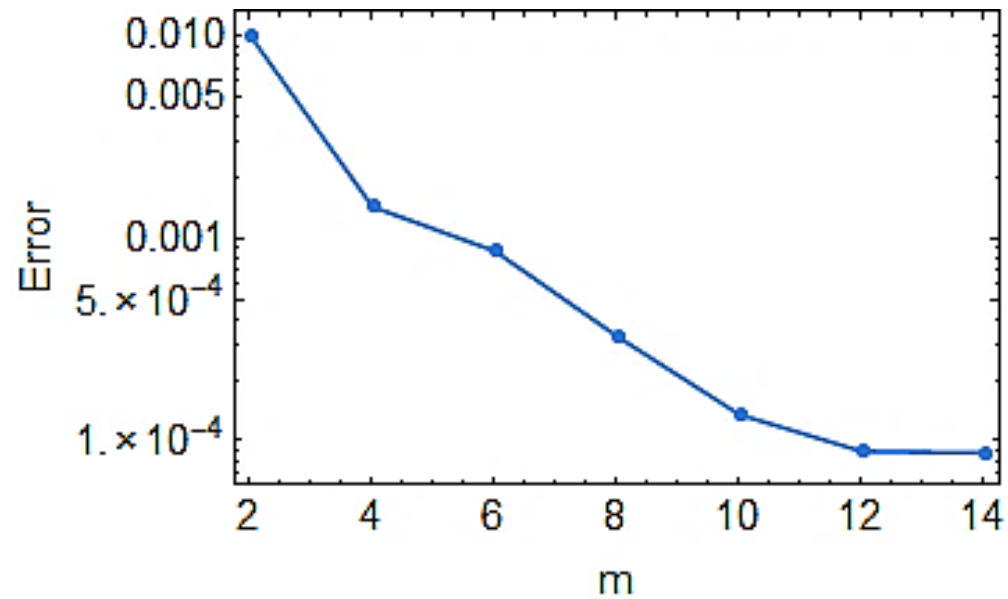

Figure 20. Error of the fluid flow problem at 15th order of HAM when $\beta=0.5, M=0.2, D a=0.5$, $R d=0.3, \operatorname{Pr}=1.0, E c=0.1, S=0.1, N b=2.0, N t=0.5, L e=1.0$, and $\lambda=0.5$.

Table 1. Comparison of the current analysis with previous studies.

\begin{tabular}{cccc}
\hline Pr & Ref. [31] & Ref. [32] & Present Values \\
\hline 1.0 & 0.9547 & 0.9547 & 0.9548 \\
2.0 & 1.4714 & 1.4714 & 1.4715 \\
3.0 & 1.8961 & 1.8961 & 1.8961 \\
\hline
\end{tabular}

Table 2. Squared residual errors using a different order of approximations when $\beta=0.1, M=0.2$, $D a=0.5, R d=0.3, \operatorname{Pr}=1.0, E c=0.1, S=0.1, N b=2.0, N t=0.5, L e=1.0$, and $\lambda=0.5$.

\begin{tabular}{ccccc}
\hline $\begin{array}{c}\text { Order of } \\
\text { Approximations }\end{array}$ & $\begin{array}{c}\text { CPU Time } \\
\text { (in Seconds) }\end{array}$ & Velocity Function & $\begin{array}{c}\text { Temperature } \\
\text { Function }\end{array}$ & $\begin{array}{c}\text { Concentration } \\
\text { Function }\end{array}$ \\
\hline 1 & 0.1404 & 0.007052 & 0.003414 & 0.000974 \\
3 & 1.6224 & 0.001049 & 0.000100 & 0.000046 \\
5 & 7.8936 & 0.000245 & 0.000018 & 0.000014 \\
7 & 22.0584 & 0.000041 & $2.1494 \times 10^{-6}$ & $4.0166 \times 10^{-6}$ \\
9 & 52.3537 & 0.000015 & $3.8766 \times 10^{-7}$ & $7.6049 \times 10^{-7}$ \\
11 & 111.166 & $2.8658 \times 10^{-6}$ & $7.1641 \times 10^{-8}$ & $1.3424 \times 10^{-8}$ \\
13 & 218.307 & $5.8343 \times 10^{-7}$ & $1.0756 \times 10^{-8}$ & $3.1915 \times 10^{-8}$ \\
15 & 324.307 & $5.8343 \times 10^{-8}$ & $1.0756 \times 10^{-8}$ & $3.1915 \times 10^{-8}$ \\
20 & 932.471 & $5.8343 \times 10^{-8}$ & $1.0756 \times 10^{-8}$ & $3.1915 \times 10^{-8}$ \\
\hline
\end{tabular}


Table 3. Numerical values of local Nusselt number via different embedded factors.

\begin{tabular}{|c|c|c|c|c|c|c|}
\hline$R d$ & $N b$ & $N t$ & $D a$ & $M$ & $E c$ & $N u_{x}$ \\
\hline 0.1 & & & & & & 0.887516 \\
\hline 0.2 & & & & & & 1.010560 \\
\hline \multirow[t]{16}{*}{0.3} & & & & & & 1.175030 \\
\hline & 0.1 & & & & & 0.957536 \\
\hline & 0.2 & & & & & 0.543257 \\
\hline & 0.3 & & & & & 0.295332 \\
\hline & & 0.1 & & & & 0.943168 \\
\hline & & 0.2 & & & & 0.558536 \\
\hline & & 0.3 & & & & 0.296429 \\
\hline & & & 0.1 & & & 0.347954 \\
\hline & & & 0.2 & & & 0.360895 \\
\hline & & & 0.3 & & & 0.386636 \\
\hline & & & & 0.1 & & 0.469674 \\
\hline & & & & 0.2 & & 0.396361 \\
\hline & & & & 0.3 & & 0.336873 \\
\hline & & & & & 0.1 & 0.465363 \\
\hline & & & & & 0.2 & 0.446376 \\
\hline & & & & & 0.3 & 0.420874 \\
\hline
\end{tabular}

Table 4. Numerical values of Sherwood number via different embedded factors.

\begin{tabular}{|c|c|c|c|c|c|c|}
\hline$R d$ & $\mathrm{Nb}$ & $N t$ & $D a$ & $M$ & $E c$ & $S h_{x}$ \\
\hline 0.1 & & & & & & 0.373181 \\
\hline 0.2 & & & & & & 0.351389 \\
\hline \multirow[t]{16}{*}{0.3} & & & & & & 0.345457 \\
\hline & 0.1 & & & & & 0.423488 \\
\hline & 0.2 & & & & & 0.409932 \\
\hline & 0.3 & & & & & 0.380207 \\
\hline & & 0.1 & & & & 0.359858 \\
\hline & & 0.2 & & & & 0.371503 \\
\hline & & 0.3 & & & & 0.405051 \\
\hline & & & 0.1 & & & 0.405019 \\
\hline & & & 0.2 & & & 0.457856 \\
\hline & & & 0.3 & & & 0.511508 \\
\hline & & & & 0.1 & & 0.358505 \\
\hline & & & & 0.2 & & 0.363773 \\
\hline & & & & 0.3 & & 0.372853 \\
\hline & & & & & 0.1 & 0.697364 \\
\hline & & & & & 0.2 & 0.664374 \\
\hline & & & & & 0.3 & 0.635633 \\
\hline
\end{tabular}

Table 5. Comparison of HAM and numerical methods for velocity function when $\beta=0.1, M=0.2$, $D a=0.5, R d=0.3, \operatorname{Pr}=1.0, E c=0.1, S=0.1, N b=2.0, N t=0.5, L e=1.0$, and $\lambda=0.16$.

\begin{tabular}{cccc}
\hline$\xi$ & HAM & Numerical & Absolute Error \\
\hline 0.0 & 0.828818 & 0.795797 & 0.033021 \\
0.5 & 0.448207 & 0.347478 & 0.100729 \\
1.0 & 0.253278 & 0.146868 & 0.106410 \\
1.5 & 0.147001 & 0.061207 & 0.085794 \\
2.0 & 0.086806 & 0.025356 & 0.061451 \\
2.5 & 0.051930 & 0.010475 & 0.041456 \\
3.0 & 0.031469 & 0.004316 & 0.027153 \\
3.5 & 0.019403 & 0.001766 & 0.017638 \\
4.0 & 0.012300 & 0.000699 & 0.011601 \\
4.5 & 0.008160 & 0.000232 & 0.007929 \\
5.0 & 0.005809 & -0.000013 & 0.005821 \\
\hline
\end{tabular}


Table 6. Comparison of HAM and numerical methods for temperature function when $\beta=0.1$, $M=0.2, D a=0.5, R d=0.3, \operatorname{Pr}=1.0, E c=0.1, S=0.1, N b=2.0, N t=0.5, L e=1.0$, and $\lambda=0.16$.

\begin{tabular}{cccc}
\hline $\boldsymbol{\xi}$ & HAM & Numerical & Absolute Error \\
\hline 0.0 & 1.000000 & 1.000000 & 0.000000 \\
0.5 & 0.861734 & 0.890063 & 0.028329 \\
1.0 & 0.742111 & 0.790668 & 0.048557 \\
1.5 & 0.322220 & 0.692580 & 0.060360 \\
2.0 & 0.528143 & 0.592819 & 0.064677 \\
2.5 & 0.428540 & 0.491288 & 0.062748 \\
3.0 & 0.333315 & 0.389007 & 0.055692 \\
3.5 & 0.242911 & 0.287353 & 0.044442 \\
4.0 & 0.157949 & 0.187751 & 0.029802 \\
4.5 & 0.079048 & 0.091556 & 0.012508 \\
5.0 & 0.006738 & $-7.975410 \times 10^{-7}$ & 0.006739 \\
\hline
\end{tabular}

Table 7. Comparison of HAM and numerical methods for concentration function when $\beta=0.1$, $M=0.2, D a=0.5, R d=0.3, \operatorname{Pr}=1.0, E c=0.1, S=0.1, N b=2.0, N t=0.5, L e=1.0$, and $\lambda=0.16$.

\begin{tabular}{cccc}
\hline$\xi$ & HAM & Numerical & Absolute Error \\
\hline 0.0 & 1.000000 & 1.000000 & $2.220450 \times 10^{-16}$ \\
0.5 & 0.833150 & 0.833913 & 0.000762 \\
1.0 & 0.694178 & 0.694714 & 0.000536 \\
1.5 & 0.574847 & 0.574322 & 0.000525 \\
2.0 & 0.470337 & 0.468678 & 0.001659 \\
2.5 & 0.377196 & 0.374759 & 0.002437 \\
3.0 & 0.292759 & 0.289950 & 0.002809 \\
3.5 & 0.214934 & 0.211942 & 0.002993 \\
4.0 & 0.142097 & 0.138718 & 0.003379 \\
4.5 & 0.073006 & 0.068553 & 0.004453 \\
5.0 & 0.006738 & $-6.874450 \times 10^{-8}$ & 0.006738 \\
\hline
\end{tabular}

\section{Conclusions}

This work describes MHD Maxwell fluid flow through an exponentially stretching sheet. The fluid's particles are immersed by a permeable surface. The flow has been analyzed with viscous dissipation, thermal radiation, Joule heating, Brownian motion, and thermophoresis phenomena. HAM has been employed for the solution of leading equations of the proposed model. The impact of numerous variables upon the flow system has been described theoretically. After an in-depth investigation of this work, the forthcoming points have been observed:

- The flow profile increases while the thermal and concentration profiles reduce with greater Darcy number.

- $\quad$ The greater Deborah number augments the velocity profile.

- The augmentation in magnetic parameter reduces the velocity profile, while the temperature and concentration profile increases with the increasing magnetic parameter.

- Both the thermal and concentration profiles are the increasing functions of the thermophoresis parameter.

- The thermal profile is the increasing function of the Brownian motion parameter and Eckert number, whereas the concentration profile is the reducing function of the Brownian motion parameter and Eckert number.

- With the augmentation in Darcy number, the permeability strength of porous media increases, which concludes the increasing conduct of thermal and mass transportation.

- In this investigation, the higher Eckert number reduces the heat and mass transfer rates.

- Growth in magnetic parameter reduces the heat transfer rate while it increases the mass transfer rate. 
- The comparison between HAM and numerical solutions ensures the validity of the solution.

Author Contributions: Conceptualization, A.D. and A.S.; methodology, A.S.; software, Z.S.; validation, S.I., formal analysis, A.D.; investigation, A.S. and Z.S.; resources, W.K.; writing-original draft preparation, A.D.; writing-review and editing, A.D., A.S., and Z.S.; supervision, S.I. and W.K.; project administration, P.K.; funding acquisition, P.K. All authors have read and agreed to the published version of the manuscript.

Funding: The financial support provided by the Center of Excellence in Theoretical and Computational Science (TaCS-CoE), KMUTT. Moreover, this research project is supported by Thailand Science Research and Innovation (TSRI) Basic Research Fund: Fiscal year 2021 under project number 64A306000005". And the APC was funded by Poom Kumam.

Institutional Review Board Statement: Not Applicable.

Informed Consent Statement: Not Applicable.

Data Availability Statement: All the supporting data are within the manuscript.

Acknowledgments: The authors acknowledge the financial support provided by the Center of Excellence in Theoretical and Computational Science (TaCS-CoE), KMUTT. Moreover, this research project is supported by Thailand Science Research and Innovation (TSRI) Basic Research Fund: Fiscal year 2021 under project number 64A306000005.

Conflicts of Interest: The authors have no conflict of interest.

\section{Nomenclature}

$\begin{array}{ll}\text { Symbol } & \text { Description } \\ (u, v) & \text { velocity components } \\ \rho & \text { Fluid density } \\ \lambda & \text { relaxation time } \\ g & \text { Force of gravity } \\ \alpha & \text { thermal diffusivity } \\ \rho c_{p} & \text { Heat capacitance } \\ D_{T} & \text { Thermophoresis coefficient } \\ T & \text { Temperature } \\ T_{\infty} & \text { Ambient temperature } \\ C_{0} & \text { Reference Concentration } \\ \beta & \text { Maxwell parameter } \\ M & \text { Magnetic parameter } \\ N b & \text { Brownian parameter } \\ D a & \text { Darcy's number } \\ S & \text { Heat source/sink parameter } \\ \operatorname{Re}_{x} & \text { Local Reynolds number } \\ B_{0} & \text { strength of magnetic effects } \\ U_{w} & \text { Stretchable velocity } \\ K & \text { Permeability } \\ c_{p} & \text { specific heat } \\ k & \text { thermal conductivity } \\ D_{B} & \text { Brownian coefficient } \\ T_{0} & \text { Reference temperature } \\ U_{0} & \text { Reference velocity } \\ C & \text { Concentration } \\ C_{\infty} & \text { Ambient concentration }\end{array}$




$\begin{array}{ll}\text { Pr } & \text { Prandtl number } \\ N t & \text { Thermophoresis parameter } \\ \text { Le } & \text { Lewis number } \\ E c & \text { Eckert number } \\ \text { Rd } & \text { Radiation parameter } \\ L & \text { Reference length }\end{array}$

\section{References}

1. Alfvén, H. Existence of Electromagnetic-Hydrodynamic Waves. Nature 1942, 150, 405-406. [CrossRef]

2. Turkyilmazoglu, M. MHD fluid flow and heat transfer due to a stretching rotating disk. Int. J. Therm. Sci. 2012, 51, 195-201. [CrossRef]

3. Kumar, K.A.; Sugunamma, V.; Sandeep, N. Effect of thermal radiation on MHD Casson fluid flow over an exponentially stretching curved sheet. J. Therm. Anal. Calorim. 2019, 140, 2377-2385. [CrossRef]

4. Khan, A.; Kumam, W.; Khan, I.; Saeed, A.; Gul, T.; Kumam, P.; Ali, I. Chemically reactive nanofluid flow past a thin moving needle with viscous dissipation, magnetic effects and hall current. PLoS ONE 2021, 16, e0249264. [CrossRef] [PubMed]

5. Khan, A.; Shah, Z.; Alzahrani, E.; Islam, S. Entropy generation and thermal analysis for rotary motion of hydromagnetic Casson nanofluid past a rotating cylinder with Joule heating effect. Int. Commun. Heat Mass Transf. 2020, 119, 104979. [CrossRef]

6. Levin, M.L.; Miller, M.A. Maxwell a treatise on electricity and magnetism. Uspekhi Fizicheskikh Nauk 1981, 135, 425-440. [CrossRef]

7. Islam, S.; Khan, A.; Kumam, P.; Alrabaiah, H.; Shah, Z.; Khan, W.; Zubair, M.; Jawad, M. Radiative mixed convection flow of maxwell nanofluid over a stretching cylinder with joule heating and heat source/sink effects. Sci. Rep. 2020, 10, 1-18. [CrossRef] [PubMed]

8. Shehzad, S.; Mabood, F.; Rauf, A.; Tlili, I. Forced convective Maxwell fluid flow through rotating disk under the thermophoretic particles motion. Int. Commun. Heat Mass Transf. 2020, 116, 104693. [CrossRef]

9. Khan, M.N.; Nadeem, S.; Ahmad, S.; Saleem, A. Mathematical analysis of heat and mass transfer in a Maxwell fluid. Proc. Inst. Mech. Eng. Part C J. Mech. Eng. Sci. 2020. [CrossRef]

10. Ahmad, M.; Muhammad, T.; Ahmad, I.; Aly, S. Time-dependent 3D flow of viscoelastic nanofluid over an unsteady stretching surface. Phys. A Stat. Mech. Appl. 2020, 551, 124004. [CrossRef]

11. Farooq, U.; Lu, D.; Munir, S.; Ramzan, M.; Suleman, M.; Hussain, S. MHD flow of Maxwell fluid with nanomaterials due to an exponentially stretching surface. Sci. Rep. 2019, 9, 1-11. [CrossRef] [PubMed]

12. Sakiadis, B.C. Boundary-layer behavior on continuous solid surfaces: I. Boundary-layer equations for two-dimensional and axisymmetric flow. AIChE J. 1961, 7, 26-28. [CrossRef]

13. Sakiadis, B.C. Boundary-layer behavior on continuous solid surfaces: III. The boundary layer on a continuous cylindrical surface. AiChE J. 1961, 7, 467-472. [CrossRef]

14. Nadeem, S.; Khan, M.; Abbas, N. Transportation of slip effects on nanomaterial micropolar fluid flow over exponentially stretching. Alex. Eng. J. 2020, 59, 3443-3450. [CrossRef]

15. Lund, L.A.; Omar, Z.; Khan, I.; Baleanu, D.; Nisar, K.S. Convective Effect on Magnetohydrodynamic (MHD) Stagnation Point Flow of Casson Fluid over a Vertical Exponentially Stretching/Shrinking Surface: Triple Solutions. Symmetry 2020, $12,1238$. [CrossRef]

16. Waini, I.; Ishak, A.; Pop, I. Mixed convection flow over an exponentially stretching/shrinking vertical surface in a hybrid nanofluid. Alex. Eng. J. 2020, 59, 1881-1891. [CrossRef]

17. Patil, P.; Kulkarni, M.; Hiremath, P. Effects of surface roughness on mixed convective nanofluid flow past an exponentially stretching permeable surface. Chin. J. Phys. 2019, 64, 203-218. [CrossRef]

18. Shah, Z.; Kumam, P.; Deebani, W. Radiative MHD Casson Nanofluid Flow with Activation energy and chemical reaction over past nonlinearly stretching surface through Entropy generation. Sci. Rep. 2020, 10, 1-14. [CrossRef]

19. Khan, M.N.; Nadeem, S.; Ullah, N.; Saleem, A. Theoretical treatment of radiative Oldroyd-B nanofluid with microorganism pass an exponentially stretching sheet. Surf. Interfaces 2020, 21, 100686. [CrossRef]

20. Manjunatha, S.; Kuttan, B.A.; Ramesh, G.K.; Gireesha, B.J.; Aly, E.H. 3D flow and heat transfer of micropolar fluid suspended with mixture of nanoparticles $(\mathrm{Ag}-\mathrm{CuO} / \mathrm{H} 2 \mathrm{O})$ driven by an exponentially stretching surface. Multidiscip. Modeling Mater. Struct. 2020, 16, 1691-1707. [CrossRef]

21. Muhammad, T.; Rafique, K.; Asma, M.; Alghamdi, M. Darcy-Forchheimer flow over an exponentially stretching curved surface with Cattaneo-Christov double diffusion. Phys. A Stat. Mech. Appl. 2020, 556, 123968. [CrossRef]

22. Waini, I.; Ishak, A.; Pop, I. Hybrid nanofluid flow towards a stagnation point on an exponentially stretching/shrinking vertical sheet with buoyancy effects. Int. J. Numer. Methods Heat Fluid Flow 2020, 31, 216-235. [CrossRef]

23. Li, Y.-X.; Alshbool, M.H.; Lv, Y.-P.; Khan, I.; Khan, M.R.; Issakhov, A. Heat and mass transfer in MHD Williamson nanofluid flow over an exponentially porous stretching surface. Case Stud. Therm. Eng. 2021, 26, 100975. [CrossRef]

24. Alazmi, B.; Vafai, K. Analysis of fluid flow and heat transfer interfacial conditions between a porous medium and a fluid layer. Int. J. Heat Mass Transf. 2001, 44, 1735-1749. [CrossRef]

25. Fatunmbi, E.O.; Ogunseye, H.A.; Sibanda, P. Magnetohydrodynamic micropolar fluid flow in a porous medium with multiple slip conditions. Int. Commun. Heat Mass Transf. 2020, 115, 104577. [CrossRef] 
26. Krishna, M.V.; Ahamad, N.A.; Chamkha, A.J. Hall and ion slip effects on unsteady MHD free convective rotating flow through a saturated porous medium over an exponential accelerated plate. Alex. Eng. J. 2020, 59, 565-577. [CrossRef]

27. Hayat, T.; Haider, F.; Alsaedi, A.; Ahmad, B. Unsteady flow of nanofluid through porous medium with variable characteristics. Int. Commun. Heat Mass Transf. 2020, 119, 104904. [CrossRef]

28. Asjad, M.I.; Ikram, M.D.; Akgül, A. Analysis of MHD viscous fluid flow through porous medium with novel power law fractional differential operator. Phys. Scr. 2020, 95, 115209. [CrossRef]

29. Khan, Z.H.; Khan, W.A.; Tang, J.; Sheremet, M.A. Entropy generation analysis of triple diffusive flow past a horizontal plate in porous medium. Chem. Eng. Sci. 2020, 228, 115980. [CrossRef]

30. Jafar, A.B.; Shafie, S.; Ullah, I. MHD radiative nanofluid flow induced by a nonlinear stretching sheet in a porous medium. Heliyon 2020, 6, e04201. [CrossRef] [PubMed]

31. Bidin, B.; Nazar, R. Numerical solution of the boundary layer flow over an exponentially stretching sheet with thermal radiation. Eur. J. Sci. Res. 2009, 33, 710-717.

32. Mukhopadhyay, S. Upper-convected maxwell fluid flow over an unsteady stretching surface embedded in porous medium subjected to suction/blowing. Z. Für Nat. A 2012, 67, 641-646. [CrossRef] 